\title{
Lusioersily
}

\section{Spiking Neural Network-based Structural Health Monitoring Hardware System}

Javed, A., Harkin, J., McDaid, LJ., \& Liu, J. (Accepted/In press). Spiking Neural Network-based Structural Health Monitoring Hardware System. In Spiking Neural Network-based Structural Health Monitoring Hardware System (pp. 1). IEEE.

Link to publication record in Ulster University Research Portal

Published in:

Spiking Neural Network-based Structural Health Monitoring Hardware System

Publication Status:

Accepted/In press: 13/10/2021

\section{Document Version}

Author Accepted version

\section{General rights}

Copyright for the publications made accessible via Ulster University's Research Portal is retained by the author(s) and / or other copyright owners and it is a condition of accessing these publications that users recognise and abide by the legal requirements associated with these rights.

\section{Take down policy}

The Research Portal is Ulster University's institutional repository that provides access to Ulster's research outputs. Every effort has been made to ensure that content in the Research Portal does not infringe any person's rights, or applicable UK laws. If you discover content in the Research Portal that you believe breaches copyright or violates any law, please contact pure-support@ulster.ac.uk. 


\title{
Spiking Neural Network-based Structural Health Monitoring Hardware System
}

\author{
Aqib Javed*, Jim Harkin, Liam McDaid and Junxiu Liu \\ School of Computing, Engineering and Intelligent Systems, \\ Ulster University, Magee Campus, Derry, Northern Ireland, United Kingdom, UK \\ *Contact: Javed-a@ulster.ac.uk
}

\begin{abstract}
Structural Health Monitoring (SHM) systems estimate damages that affect structural reliability. Modern SHM requires continuous monitoring to detect damage caused by day-to-day loading and usage. SHM analyzes structural integrity and identifies weaknesses leading to potential building collapse. In disaster situations e.g., earthquakes, SHM systems enable early assessment of building safety and therefore ensure evacuation and prevention of human losses. In an urban area, multi-story constructions in congested areas become a hazard if their structural health is not well monitored and maintained properly. A key challenge is the ability to detect damages in an efficient manner for edge computing. In this work, we propose an SNN based low-cost, energy-efficient, and standalone damage classification model for SHM. The proposed classification model is implemented on FPGAs and results show a classification accuracy of $99.46 \%$ on a sensory dataset for earthquake damage on a 7-Story concrete building.
\end{abstract}

Keywords- FPGAs, Structural Health Monitoring; Spiking Neural Networks; Earthquake.

\section{INTRODUCTION}

The United Nation's report on Disaster Risk Reduction (UNDRR) [1] shows an increasing trend in the number of natural disasters (fire, earthquake, flood etc) over the last several decades. Urbanization triggered by the $19^{\text {th }}$ century industrial revolution brought economic welfare to the society [2]. Rising migration and population has allowed cities to construct complex and tall megastructures to accommodate increasing residential demands as well as enrich the city landscape [3]. The life span of typical building lasts for decades but regular maintenance helps to improve structural health and life span of important buildings for centuries. Cities hosts large portion of population and plays an important role in cultural, financial, social, ecological and geopolitical aspects of our societies [4]. Any natural disaster like earthquake, hurricane, floods etc. in these densely populated areas is catastrophic for the local civilization [5]. Megacities constructed close to the tectonic fault-lines are more susceptible to devastation by earthquakes in the near future [6]. Vibrational movements caused by earthquake shake the building by exerting stress at critical structural points causing fractures and collapse [7]. Despite modern technology advances and the usage of sensory equipment's when an earthquake will occur is unpredictable. The only reliable source to determine its future occurrence is to analyze previous seismic data along with geological formation of a particular area. Governments have invested in the critical infrastructures i.e., hospitals, schools, military bases, bridges, dams, and emergency shelters to confront impact of unprecedented natural disasters. The structural heath of these critical infrastructures must be check periodically to validate their endurance, reliability, and usability in any emergency [8]. Traditional method requires personal assessments by experienced inspectors along with portable sensory equipment. These costly manual assessments can be done periodically (quarterly, biannually, or annually) as it requires dedicated high-spec equipment that further ads in costs [9].

Modern SHM techniques equipped with complex software algorithms that processes real-time structural information to automatically diagnose structural changes and damages caused by the aging, erosion, and regular load [10]. The recent trend of equipping national, cultural, residential, and commercial building with an efficient SHM systems, is to evaluate damages and aid in reducing any impact from potential human-made or natural disasters. [11]. The SHM system installed in the building provides real-time information about damages caused by the natural disaster or day-to-day work loads thus providing decision making information about its safety and usage. The sensory data information can be monitored and processed onsite or through remotely locations via internet or other communication channels. The success of modern real-time SHM systems relies broadly on the authenticity, capability, and efficiency of the decision-making information processing software algorithms. Ideally these algorithms must be adaptive to all structural changes caused by damages as well as regular repairs. Machine learning algorithms i.e., Neural Networks (NNs) have shown significant benefit in detection and classification. Especially, 3rd generation neural model i.e., Spiking Neural Networks (SNNs) are more biological realistic mathematical models that offers an alternative and efficient event-based computing. Recent study shows that digital SNNs are computationally powerful and require less hardware area as compare to an ANN [12]-[14]. In this work propose a hardware implementation of an SNN based SHM system to analyze and classify structural damages caused by earthquakes. The structural damage classification performance of the proposed model is evaluated on a sensory dataset generated by execution of four historical earthquake ground motions on a full-scale 7story concrete building.

Section II provides background on existing damage classification techniques for SHM systems. Section III explains hardware implementation of proposed classification model and section IV provides experimental setup to analyse performance of SNN-based classifier. Section V presents simulation results 
and analysis with existing models. Section VI concludes this research and outlines future work.

\section{BACKGROUND AND LITERATURE REVIEW}

Rapid urbanization was triggered by the eighteenth-century industrial revolution that caused massive relocations and the migration of peoples towards important trade routes and strategic locations such as near seaports, ore or mineral mines, or industrial areas etc. [3]. These migrations have not only improved socioeconomic condition of local area but also provides an opportunity to multi-cultural society to develop complex building structures with newer construction material and techniques [2]. Local and central government have invested in upscaling human development in urban areas by providing physical infrastructures (landscape building, libraries, railways, roads, business parks, schools, electricity, and other day-to-day utilities) and critical infrastructure (hospital, military bases, emergency shelter) [15]. These public buildings and infrastructure were designed and construct with the resources available at the time of construction to fitful their long-term usability by withstanding extreme weather, capacity, and load. The use of concrete in the construction of buildings and bridges is the foremost reason for the existence of many 19th century iconic building across the world [16]. These building requires regular structural health monitoring to endure their usage, health, and robustness. The constructed infrastructure is not only threatened to decay with aging and corrosion but also exposed to extreme events (earthquake, Tsunami, floods, hurricane etc.).

There are over 457 volcanoes within $100 \mathrm{Km}$ range of megacities (cities with pop above 1 million) across the world [17]. Due to urbanization, environmental degradation and poor infrastructure conditions of commercial and residential buildings makes these areas more prone to natural catastrophe [6]. The consequences of an earthquake are not limited to the instant when it has occurred, but they are also accompanied by series of events i.e., landslide, flooding, collapse of power and transportation infrastructure etc. which can lasts for years [6], [18]. The 1999 Chi-Chi earthquake triggered 10,000 landslides across mountainous terrain of Central Taiwan [19]. These aftermath events not only restrict emergency response from outside the disaster area but also slowed down the rehabilitation process in the affected areas. The demand in curbing of the socioeconomic effects associated with these natural disasters has encouraged the governments to implement standardized policies to make infrastructure and communities more durable and robust [18].

Therefore structural health of the critical infrastructures i.e., hospital, military bases and emergency shelters should meet the required safety, reliability, and useability standards in the disaster-affected areas [20]. These front-line critical infrastructures can assist in emergency situations by providing instant medical aid, shelter, and human resource to counter consequences of natural disaster. The earthquake can produce extreme stress causing cracks and deformation at critical structural points which ultimately cause collapse of infrastructure [11]. The unseen/hidden structural damages should be assessed by an efficient structural health monitoring system before using critical buildings for emergency response.

SHM systems follows four discrete steps: signal acquisition, signal processing, feature extraction and damage classification to assess reliability of any structures. The number of sensors i.e., piezoelectric accelerometers with orthogonal axis of acceleration or thermometer [21], wavelet [22], ultrasonic wave sensor [23], fiber optic sensor [4] etc. are attached at critical points of the building to generate structural information i.e., acceleration, velocity, displacement etc. to the connected computational environment [24]. This sensory analogue information is digitized with the help of different Analogue to Digital Converters (ADC) supported by signal processing tools. Feature extraction and identification methods relies on empirical data to differentiate between active features formed by the structural changes and momentary noise signals generated by the sensors [9]. SHM requires complex feature extraction methods i.e., orthogonal decomposition method to extract dynamic sparse features caused by the vibrational motion of the structure[9]. Once important featured signals are extracted from the sensory signal, a classification algorithm is used to analyze and categorize damages [25]. The strength of an SHM system depends on the accuracy of its classifier. Early SHM used K-Mean (KM) clustering techniques to classify structural damages into number of categories, however the KM technique is sensitive to the input data and sometimes cluster requires manual starting point. Other existing classification techniques include wavelet-based approaches, Kalman filterbased methods and edge detection method for SHM [22]. NNs have outperformed existing classification methods in terms of classification accuracy and precision. NNs requires dataset of varying feature patterns to adjust their synaptic weights during the training phase. Recent adaptation of ANNs to estimate strain responses of building columns under windy condition has paved the way for NNs deployment in SHM systems [5]. These NNs are complex mathematical models that requires advance hardware system to execute computationally intensive equations. To enjoy high performance parallel computing with customized hardware implementation on programmable architecture has urged researcher to explore FPGA based SHM systems to boost data processing, feature extraction and classification for SHM systems. Number of feature extraction techniques including Fast Fourier Transform (FFT) [26], Hilbert-Huang Transform (HHT) [27], Bayesian statistics [28], non-linear time series analysis [29] and Discrete Wavelet Transform (DWT) to record acoustic emission are implemented on FPGA to analyze and classify sensory/imagery signals data to aid SHM systems. These classifiers require mapping of complex mathematical (differential) equations on FPGA thus incur hardware area and power. Therefore, a need of an efficient (low-cost) SHM classifier is imminent demand to enhance performance of existing SHMs during unwanted situations.

The advancement in high processing power on-chip processors has brought life to the hardware implementation of SNNs. A recent study has shown SNNs as an efficient and powerful classification technique for SHM [9]. Contrary to conventional artificial neurons, spiking neurons are spike- 




Fig. 1 Proposed SNN based classifier for SHM system.

event-driven that utilize energy to process information only at the arrival of spike events at the input to neurons. This eventbased discrete phenomenon of spiking neurons makes them more energy efficient and appropriate for hardware implementations. Therefore, this work proposes an FPGAbased low-cost SNN classifier to enhance performance and lifespan of SHM systems.

\section{SHM HARDWARE CLASSIFIER}

This section outlines the hardware architecture of proposed classifier to detect structural damages in critical buildings during disaster situation.

\section{A. SNN Based SHM Classification Model}

The SHM systems capture continuous data of structural variations caused by the vibrational motions at key building points. The raw sensory data is often transmitted to a central SHM system via a wired or wireless medium [30]. Modern SHM digitize sensory information to extract feature required for structural damage classification. The digital features are transformed into temporal patterns before sending it to a classifier. In this work we proposed hardware implementation of an SNN-based structural health monitoring model to classify structural damage.

SNNs process and encode information in the temporal domain by dealing with time of spike instead of amplitude of neural spike. The temporal patterns generated during the feature extraction process can be directly fed into an SNN for training and validation. A conductance-based Hodgkin-Huxley neuron model is the most realistic mathematical model that requires high hardware resources to solve non-linear differential equations [31], whereas the Integrate and Fire (IF) model is a more simplified mathematical spiking model of biological neurons [32]. Leaky integrate and Fire (LIF) neuron model is the modified version of the IF model with the Hodgkin-Huxley neuron model-type leaky neural membrane [33]. The LIF neuron provides right trade-off between reduced hardware area and sufficient spiking performance [34]. This work proposes to use the LIF neuron model in the SNN classifier. The mathematical model of the LIF neuron can be expressed as

$$
\tau_{m} \frac{d\left(u_{i}\right)}{d t}=-u_{i}(t)+R I_{i}^{s y n}(t)
$$

Where $I_{i}^{s y n}(t)$ is synaptic current from presynaptic neurons at time $(t), \tau_{m}$ is the membrane time constant of decaying membrane potential in the absence of spikes, and $R$ is the neural resistance. Synaptic current $I_{i}^{\text {syn }}(t)$ is modelled by

$$
I_{i}^{s y n}(t)=\left\{\begin{array}{c}
w_{j}\left(e^{\left(\frac{T-t}{\tau}\right)}-e^{\left(\frac{T-t}{4 \tau}\right)}\right) \text { if }(T-t)>0 \\
0 \quad \text { if }(T-t) \leq 0
\end{array}\right.
$$

where $w_{j}$ is the weight of $j_{t h}$ synapse towards post-synaptic neuron $i$ at time $t$. $T$ is the spike train time, $\tau$ is the synaptic current time constant.

The proposed classification model is supported with spike-prop as a learning algorithm for synaptic weight updates during training. Bothe's spike-prop is an efficient and reliable leaning model to adjust synaptic weights when dealing with temporal patterns [35]. Spike-prop updates the cost function $(E)$ while minimizing Mean Square Error (MSE) between actual $t_{j}^{a}$ and desired $t_{j}^{d}$ spike time using

$$
E=\frac{1}{2} \sum_{j \in J}\left(t_{j}^{a}-t_{j}^{d}\right)^{2}
$$

\section{B. Hardware Architecture}

The proposed SNN based structural health classifier comprising up of spike generator, LIF neuron based SNN model and spike analyzer. Fig. 1 shows hardware architecture of proposed LIF based classifier for SHM system to analyze structural health, integrity, and reliability. In the feedforward neuron model, each presynaptic LIF neuron generates synaptic current for the post synaptic neuron. LIF neuron requires temporal input, synaptic weights (generated during training) to accurately classify structural health.

The sensors attached to the structure generates floating point value thus a Spike Generator is integrated before SNN input layer to convert sensory values into temporal fixed-point values. A running mean/average algorithm is used to normalize incoming sensory values into temporal spike patterns. The spike generator continuously updates mean value to keep input spike within defined spike time frame $T$ ( $T_{\min }$ and $\left.T_{\max }\right)$. Fig. 2 illustrates working principle of LIF neuron hardware components developed to analyze proposed classifier for SHM 
system. These temporal input spikes are fed into SNN to classify structural health by spiking at different damaged classification times. Neural synapse generator is implemented to generate synapse for each postsynaptic neuron in the SNN based classifier (as shown in Fig. 2). Each LIF neuron is connected to number of post-synaptic neurons and receives multiple synapses from pre-synaptic neurons where each synapse generates its own synaptic current $I_{i}^{\text {syn }}(t)$ towards post-synaptic neuron $i$ arriving at separate time $t$ using equation (2). A Synaptic current module is used to gather all incoming weighted and delayed synapses into a unified synaptic current array sized [1x400] for LIF neuron (shown in Fig. 2). An array of trained weights $[N x S]$ is stored inside neuron to generate weighted synaptic current for LIF, where the size of weight array depends on number of pre-synaptic neurons $N$ and number of synapses $S$ from each pre-synaptic neuron. This model assumed 10 synaptic connections between each presynaptic and post-synaptic neuron generated using delay module shown in Fig. 2.



Fig 2. LIF neuron architecture for spike generation and propagation

The LIF neuron has a leaky membrane potential $\left(V_{m}\right)$ that fluctuates between minimum voltage $\left(V_{\min }=-70 \mathrm{mV}\right)$ and threshold voltage $\left(V_{t h}=-35 \mathrm{mV}\right)$. On arrival of each synaptic current, the membrane potential $\left(V_{m}\right)$ increases towards threshold voltage $\left(V_{t h}\right)$ whereas in absence of spike membrane potential decreases towards minimum membrane voltage $\left(V_{\text {min }}\right)$. A Membrane voltage generator and comparator is employed using equation (1) to calculate membrane voltage on arrival of each synaptic input. The LIF neuron membrane potential is fed to voltage comparator (as shown in Fig. 2) to continuously compare it with pre-defined threshold voltage $\left(V_{t h}=-35 \mathrm{mV}\right)$, if membrane voltage surpasses threshold voltage $\left(V_{m}>V_{t h}\right)$, voltage comparator sends a signal to spike generator to fire a neural sike and record time of spike before resetting membrane potential to reset voltage $\left(V_{\text {reset }}=\right.$ $-75 \mathrm{mV})$. Once neuron spikes, spiking neurons go through mandatory refractory time $\left(R_{t}=10\right.$ clock cycles $)$ that allow LIF neurons to resettle membrane potential at $\left(V_{\min }\right)$ and get ready to accept next synaptic input. The time of spike of output layer neuron is forwarded to Spike analyzer to classify structural health of the building. The proposed classification model is trained to classify structural health with respect to predefined spike time $(16,18,20$ and 22) for four damage levels (S0, S1, S2 and S3) with $1 \%$ tolerance. The output neuron spike time is compared with predefined structural health level that reflects grade of damages in the building. The classification output can be forwarded to SHM system to take appropriate actions.

\section{Neural Model Training}

Neural networks require an efficient learning model to update synaptic weights in order to maximize classification accuracy. This work considered Spikeprop, a supervised learning model for spiking neurons that encodes information by adjusting synaptic weight based on the time of first spike and propagate neural spike from presynaptic layer neurons to postsynaptic layer neurons [35]. Comparably to ANN's backpropagation learning model, Spikeprop adjusts synaptic weights to bring output layer neurons spike time close to desire spike time at the end of each iteration. Contrary to rate/phase encoding, the main advantage of SpikeProp is encoding of structural health information at each sensory spiking input. The SNN model is trained before the deployment of hardware system, and this was done using historical sensory data. Once the model is trained, the generated synaptic weights can be used for the hardware system.

\section{SNN Based Classifier}

The proposed SNN based structural health classifier is able to connect directly with the sensors to read real-time data and perform damage classification. The hardware SNN provides parallel processing thus enables multiple hardware architectures to process sensory data concurrently. Furthermore, these devices operate with low-power DC requirements and during power failure in disastrous situation they can be backup with battery pack or solar power to continuously work onsite [26]. Therefore, a single FPGA installed on critical infrastructure will be able to monitor realtime structural health. This configuration helps the proposed hardware to work standalone in disaster affected structures i.e., building, bridges, dam where human access can be fatal.

Fig. 2 shows an overview of FPGA hardware blocks for the SHM system classifier. The raw sensory input is first normalized with average running mean value to generate temporal fix-point number for spiking neural hardware compatibility. The structure is fed with 45 accelerometer sensors therefore we proposed each sensory output data is normalized independently to its own running mean value. During SNN training, number of network topologies (including number of neural layers, number of neurons in each layer, and number synaptic connections between two feed-forwarded connected neurons) are evaluated to identify compact SNN size without compromising classification performance. Fig.2 illustrates a 3-layer fully connected SNN implementation on FPGA, where input layer has 45 neurons (1:1 ratio with number of sensors) to read normalized sensory input, 10 hidden layers neurons for information encoding and 1 neuron in output layer to determine structural health and damage level. A single 
spiking neuron at output layer can be trained to classify number of discrete states by varying spike firing time. The time of spike of output neuron determine structural health and suggest usability and reliability of under observation structure. The classification output can be utilized by external hardware or SHM systems to take appropriate actions i.e., warning, closure of bridge, building or dam etc.

\section{EXPERIMENTAL SETUP}

This section explains the experimental setup established to implement the proposed SNN based fine-grain level congestion prediction models.

\section{A. Earthquake Data Acquisition}

The effectiveness of SHM can be determined by installing sensing devices at critical points of the building to measure accuracy of its response on structural damage classification. This work has considered a full-scale 7-story structure consisting of up of four gravity columns for stability, main wall and back wall on each floor, a concrete slab to separate floors levels and an auxiliary positioned column [7]. The structure was equipped with sensors (45 accelerometers) to transmit data signals using a nine-node distributed data acquisition system. Between October 2005 to January 2006, a sequence of 68 dynamic tests (ambient vibration tests, free vibration tests, and forced vibration tests) were applied using the UCSD-NEES shake table [7]. The structure also undergoes vibratory motions to replicate four historical earthquakes records: traversal and longitudinal components of 1971 San Fernando earthquake $(\mathrm{Mw}=6.6, \mathrm{Mw}=6.6)$ and longitudinal and 360" component of 1994 Northridge earthquake $(\mathrm{Mw}=6.7, \mathrm{Mw}=6.7)$. The building undergoes several structural damages during intense and realistic earthquake. To minimize reading error, the structure was subjected to white noise (WN) base extraction test run for 8 min followed by $3 \mathrm{~min}$ long ambient vibration (AV) test to check the instrumentation and data acquisition system used in experimentation. The damages recorded in the structure are classified in four structural damage state: S0, S1, S2 and S3. Where S0 is defined as the undamaged, S1, S2 and S3 are damage caused by the first, second and third artificially generated earthquake events respectively (as shown in Table 1).

The raw experimental data samples extracted from the sensors are translated to generate mean value samples for structural heath classification. The featured temporal dataset can be used for training and validation of proposed SNN based SHM classifier.

TABLE 1

\section{DAMAGE CLASSIFICATION}

\begin{tabular}{|c|c|}
\hline Damage state & Test description \\
\hline State-0 (S0) & $\begin{array}{c}8 \text { min white noise base excitation process \& } 3 \text { min } \\
\text { ambient vibration }\end{array}$ \\
\hline State-1 (S1) & $\begin{array}{c}\text { After the } 1 \text { st earthquake excitation, with } 8 \text { min } \\
\text { white noise base excitation process \& } 3 \text { min } \\
\text { ambient vibration }\end{array}$ \\
\hline State-2 (S2) & $\begin{array}{l}\text { After the } 2 \text { nd earthquake excitation, with } 8 \mathrm{~min} \\
\text { white noise base excitation process \& } 3 \mathrm{~min} \\
\text { ambient vibration }\end{array}$ \\
\hline State-3 (S3) & $\begin{array}{c}\text { After the 3rd earthquake excitation, with } 8 \mathrm{~min} \\
\text { white noise base excitation process \& } 3 \mathrm{~min} \\
\text { ambient vibration }\end{array}$ \\
\hline
\end{tabular}

\section{B. Simulation Setup}

To validate classification performance, a three layered fully connected SNN was designed and implemented on FPGA using VHDL. The proposed SNN used LIF based neurons with SpikeProp as a learning algorithm to adjust synaptic weights during training phase.

The fully connected, feed forwarded SNN has 45 neurons in the input layer to obtain data from 45 accelerometer sensors, 10 neurons in the hidden layer for data encoding and one neuron in the output layer to classify damage with spike time. Fig 3 shows an output layer neuron spike time range used to encode structural health using sensory data to classify structural damage. If the output neuron fire spike at $16 \pm 1 \%$ then structure is classified at $\mathrm{S} 0$ state, else if output neuron spiked at $18 \pm 1 \%$ then structure has State-1 damages from an earthquake, else if output neuron generate spike at $20 \pm 1 \%$ then structure is state3 level damages from two earthquakes, else if neuron spike at $22 \pm 1 \%$ then structure is in critical condition (State-4) after surviving three artificially induced earthquakes. Fig 3 shows that for an incoming sensory input data, the output layer neuron of SHM-SNN spiked at $20 \pm 1 \%$ range therefore the SHM system classifies under-observation building as state-3 level damage structure caused by two earthquakes.

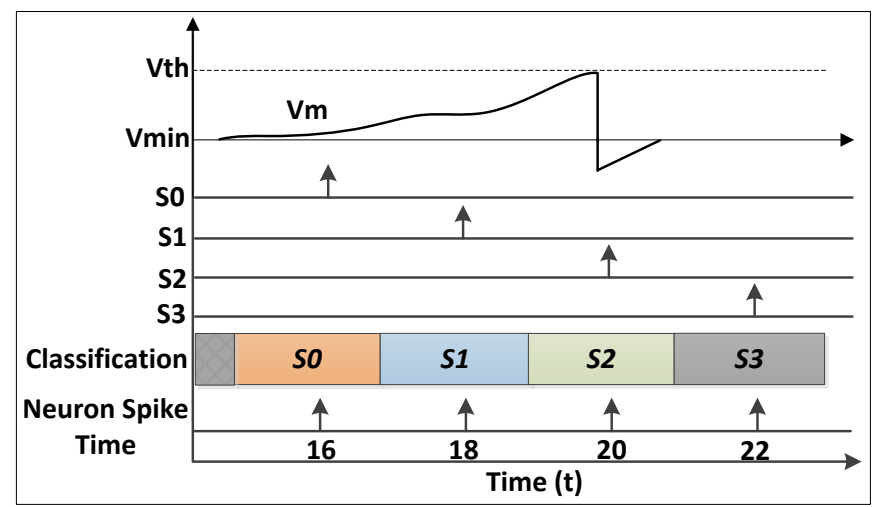

Fig. 3: Timing diagram of structural health classifier

The featured temporal mean value samples are fed directly into the SNN for training and testing. The model is trained on $70 \%$ random dataset and was simulated for 2,000 epochs to adjust synaptic weights to achieve $<1 \%$ MSE. Once trained, proposed classification model was fed with $30 \%$ unseen dataset to analyze the classification of structural health according to damage states (S0, S1, S2 and S3). The success of proposed model relies on how accurately it classifies damages using mean valued feature dataset.

The model is analyzed against prediction accuracy $\left(P_{a}\right)$, model Sensitivity $\left(P_{s e}\right)$ and Precision $\left(P_{p}\right)$ on mean valued dataset generated by the vibrational motion of under observation sevenstory structure.

$$
\begin{gathered}
P_{a}=\frac{\left(\sum T P+\sum T N\right)}{S} \\
P_{s e}=\frac{\left(\sum T N\right)}{S} \\
P_{p}=\frac{\left(\sum T P\right)}{S}
\end{gathered}
$$


where correct classification is termed as True Positive (TP), incorrect classifications are labelled as True Negative (TN) and $(S)$ is total sample dataset size used for analysis.

\section{RESUlts AND ANALYSIS}

\section{A. Simulation Analysis}

Simulation results shows that proposed SNN based structural health classifier has correctly identified damages in the 7-story concrete structure with an accuracy of $99.46 \%$. Table 2 shows classification accuracy for each damage category. It is depicted that the damage levels S0, S1, S2 and S3 are classified with $100 \%, 99.90 \%, 99 \%$. $100 \%$ and $99.61 \%$ accuracy using proposed SHM system.

TABLE 2 CLASSIFICAITON ACCURACY

\begin{tabular}{|c|c|c|c|c|}
\hline Predict $^{\text {Actual }}$ & S0 & S1 & S2 & S3 \\
\hline S0 & $\mathbf{9 9 . 6 8 \%}$ & $0.32 \%$ & 0 & 0 \\
\hline S1 & $1 \%$ & $\mathbf{9 9 \%}$ & 0 & 0 \\
\hline S2 & 0 & 0 & $\mathbf{1 0 0} \%$ & 0 \\
\hline S3 & $0.38 \%$ & 0 & 0 & $\mathbf{9 9 . 6 2} \%$ \\
\hline
\end{tabular}

Table 3 shows that proposed model is able to detect and classify structural damage with $99.46 \%$ accuracy. Furthermore, proposed SNN classifier has shown classification sensitivity and classification precision of $99.9 \%$. The classification sensitivity and precision determine the ability of proposed classification model to correctly identify and classify structural health damages across all damage categories.

TABLE 3 SHM CLASSIFICATION PERFORMANCE

\begin{tabular}{|c|c|c|c|}
\hline & Accuracy & Sensitivity & Precision \\
\hline Average & $99.46 \%$ & $99.9 \%$ & $99.9 \%$ \\
\hline
\end{tabular}

\section{B. Hardware analysis:}

The proposed model is implemented using the Xilinx Artix-7 (xc7a200t ffv1156-1L) FPGA board. The FPGA performs multiple operations in parallel to provide real-time solution at a low hardware and power cost [36]. In an event of natural disasters, the power loss across an area restricts computer backed SHM system to generate and send structural health report for damage classification and analysis. The FPGA devices utilize low power and can be backed up with portable batteries or solar power to classify sensory data and send structural health report to remote server/operation unit thus allowing to perform systematic analysis without endangering human lives [21].

TABLE 4 HARDWARE OVERHEAD

\begin{tabular}{|c|c|c|c|}
\hline Resource & Estimation & Available & Utilization (\%) \\
\hline LUT & 72403 & 303600 & 23.848156 \\
\hline FF & 94606 & 607200 & 15.580699 \\
\hline DSP & 6 & 2800 & 0.2142857 \\
\hline
\end{tabular}

Table 4 outlines that the proposed classification model (45 input, 10 hidden-layer and one output neurons) requires $23.85 \%$ of Look-Up-Table (LUT), $15.58 \%$ Flipflop (FF) and $0.21 \%$ of DSP resources available on Xilinx Artix-7 board.

\section{Comparison to Existing Techniques:}

This work implemented a damage classifier to process data of all accelerometer sensors installed at 7-story building to observe and record vibrational motion caused by the earthquake. The existing low-cost FPGA based classifiers for SHM has proposed an exclusive FPGA for each sensor to perform signal processing, filtration, and feature extraction processes on sensory data. Table 5 illustrates an estimated hardware requirement for Discrete Wavelet Transform (DWT) [37] and Hilbert Transform (HT) [29] based damage classifier for 7-story concrete structure. It is evident that SNN-classifier requires $78.65 \%$ less Look-Up-Table (LUTs), $77.33 \%$ less Flip-flops (FFs) and $87.33 \%$ (static and dynamic) power saving at $5 \mathrm{MHz}$ operating frequency when compared to DWTclassifier. Moreover, proposed classification model has saved $88.40 \%$ LUTs and $85.79 \%$ FFs hardware resources as compared to HT-classifier for SHM system. Therefore, SNNbased classifier is a low-cost solution for SHM system with the structural damage classification accuracy of $99.48 \%$ on earthquake dataset.

\section{TABLE 5}

\section{HARDWARE OVERHEAD COMPARISON}

\begin{tabular}{|c|c|c|c|c|}
\hline Technique & $\begin{array}{c}\text { Discrete Wavelet } \\
\text { Transform } \\
\text { (DWT) [37] }\end{array}$ & $\begin{array}{c}\text { Hilbert } \\
\text { Transform } \\
\text { (HT) [29] }\end{array}$ & Proposed & Available \\
\hline Resource & 339120 & 623925 & 72403 & 303600 \\
\hline FF & 417330 & 665685 & 94606 & 607200 \\
\hline Power & $60 \mathrm{~W}$ & N/A & $5.27 \mathrm{~W}$ & N/A \\
\hline
\end{tabular}

\section{CONCLUSION}

The SHM system requires robust classifier that helps in identification of structural damages that may affect structural integrity of building. This work proposed hardware implementation of SNN based damage classifier to enhance efficacy of SHM system. The proposed model is implemented on Artix-7 FPGA board and requires fraction of hardware resources to detect structural damages with caused by earthquake $99.46 \%$ accuracy, $99.9 \%$ sensitivity and $99.9 \%$ precision. Furthermore, the SNN-based classifier for SHM system requires least hardware resources as compared to existing state-of-the-art DWT-based classifier and HT-based classifier for SHM systems. The FPGA based models are power efficient and able to analyse structural health in extreme disastrous conditions without compromising health monitoring performance [26]. Future work includes deployment of FPGA based SNN on critical structures including bridges, hospital, school and community hall buildings to monitor damage performance on day-to-day load as well as under disaster condition.

\section{REFERENCES}

[1] U. N. O. for UNDRR (2019), Global Assessment Report on Disaster Risk Reduction, Geneva, Switzerland and D. R. R. (UNDRR)., Global Assessment Report on Disaster Risk Reduction.

[2] S. Li and Y. Ma, "Urbanization, Economic Development and Environmental Change," sustainability, no. 6, pp. 5143-5161, 2014.

[3] M. Antrop, "Landscape change and the urbanization process in Europe," Landsc. Urban Plan., vol. 67, no. 1-4, pp. 9-26, 2004.

[4] J. M. López-higuera, S. Member, L. R. Cobo, A. Q. Incera, and A. Cobo, "Fiber Optic Sensors in Structural Health Monitoring," J. Light. Technol., vol. 29, no. 4, pp. 587-608, 2011.

[5] B. K. Oh, K. J. Kim, Y. Kim, H. S. Park, and H. Adeli, "Evolutionary 
learning based sustainable strain sensing model for structural health monitoring of high-rise buildings," Appl. Soft Comput. J., 2017.

[6] C. Chen and T. Cheng-wu, "Natural disaster management mechanisms for probabilistic earthquake loss," Nat. Hazards, vol. 60, no. 1, pp. 1055-1063, 2012.

[7] B. Moaveni, X. He, J. P. Conte, J. I. Restrepo, and M. Panagiotou, "System identification study of a 7-story full-scale building slice tested on the UCSD-NEES shake table," J. Struct. Eng., vol. 137, no. 6, 2011.

[8] A. E. Bouzenad et al., "A semi-supervised based k-means algorithm for optimal guided waves structural health monitoring: A case study," Inventions, 2019

[9] L. Pang et al., "Case Study - Spiking Neural Network Hardware System for Structural Health Monitoring," Sensors, vol. 20, no. 18, p. 5126, 2020.

[10] V. Gi and M. D. Trifunac, "A note on predetermined earthquake damage scenarios for structural health monitoring," Struct. Control Heal. Monit., vol. 19, no. June 2011, pp. 746-757, 2012.

[11] M. Roghaei and A. Zabihollah, "An Efficient and Reliable Structural Health Monitoring System for Buildings after Earthquake," in Procedia - Social and Behavioral Sciences, 5th International Conference on Chemical, Biological and Environmental Engineering - ICBEE 2013 \& 2nd International Conference on Civil Engineering - ICCEN 2013, 2014, vol. 9, pp. 309-316.

[12] X. Wang, X. Lin, and X. Dang, "Supervised learning in spiking neural networks : A review of algorithms and evaluations," Neural Networks, vol. 125, no. 2020 Special Issue, pp. 258-280, 2020.

[13] J. H. Lee, T. Delbruck, and M. Pfeiffer, "Training Deep Spiking Neural Networks using Backpropagation," CoRR, vol. abs/1, pp. 110.

[14] A. Javed, J. Harkin, L. Mcdaid, and J. Liu, "Exploring Spiking Neural Networks for Prediction of Traffic Congestion in Networks-onChip," in IEEE International Symposium on Circuits and Systems (ISCAS), Seville Spain 2020 (accepted), 2020.

[15] X. Wang and T. C. Hutchinson, "Evolution of modal characteristics of a mid-rise cold-formed steel building during construction and earthquake testing," Earthq. Engng Struct Dyn, vol. 49, no. June, pp. 1539-1558, 2020.

[16] G. Song, C. Wang, and B. Wang, "Structural Health Monitoring ( SHM ) of Civil Structures," Appl. Sci., vol. 7, pp. 3-5, 2017.

[17] D. Paton, L. Smith, M. Daly, and D. Johnston, "Risk perception and volcanic hazard mitigation: Individual and social perspectives," $J$. Volcanol. Geotherm. Res., vol. 172, pp. 179-188, 2008.

[18] S. E. Chang, "Transportation Performance, Disaster Vulnerability , and Long-Term Effects of Earthquakes," in Second EuroConference on Global Change and Catastrophe Risk Management, 2000, pp. 112.

[19] B. Khazai and N. Sitar, "Evaluation of factors controlling earthquakeinduced landslides caused by Chi-Chi earthquake and comparison with the Northridge and Loma Prieta events," Eng. Geol., vol. 71, pp. 79-95, 2003.

[20] J. Ou and H. Li, "Structural Health Monitoring in mainland China : Review and Future Trends," Struct. Heal. Monit., vol. 9, no. 3, pp. 219-231, 2010.

[21] J. F. Wang, Z. Y. Xu, X. L. Fan, and J. P. Lin, "Thermal Effects on Curved Steel Box Girder Bridges and Their Countermeasures," J. Perform. Constr. Facil., vol. 31, no. 2, 2017.
[22] Z. Hou, A. Hera, and A. Shinde, "Wavelet-Based Structural Health Monitoring of Earthquake Excited Structures," Comput. Civ. Infrastruct. Eng., vol. 21, pp. 268-279, 2006.

[23] M. Abbas and M. Shafiee, "Structural Health Monitoring ( SHM ) and Determination of Surface Defects in Large," Sensors, vol. 18, no. 11,2018

[24] S. Sony, S. Laventure, and A. Sadhu, "A literature review of next generation smart sensing technology in structural health monitoring," Struct. Control Heal. Monit., vol. 26, no. 3, pp. 1-22, 2019.

[25] S. Eftekhar Azam, A. Rageh, and D. Linzell, "Damage detection in structural systems utilizing artificial neural networks and proper orthogonal decomposition," Struct. Control Heal. Monit., 2019.

[26] M. Azarbayejani, M. Jalalpour, and M. M. R. Taha, "Field application of smart SHM using field programmable gate array technology to monitor an RC bridge in New Mexico," Smart Mater. Struct., vol. 20, no. 8, 2011.

[27] M. Hedley et al., "Sensor network for structural health monitoring," in Proceedings of the 2004 Intelligent Sensors, Sensor Networks and Information Processing Conference, 2004, Melbourne, VIC, Australia, 2004, pp. 361-366.

[28] C. Grosse, G. Mclaskey, S. Bachmaier, S. D. Glaser, and M. Krüger, "A hybrid wireless sensor network for acoustic emission testing in SHM," Proc. SPIE 6932, Sensors Smart Struct. Technol. Civil, Mech. Aerosp. Syst. 2008, 693238, vol. 6932, 2008.

[29] J. D. Jones, S. Member, and J. Pei, "Embedded Algorithms Within an FPGA to Classify Nonlinear Single-Degree-of-Freedom Systems," IEEE Sens. J., vol. 9, no. 11, pp. 1486-1493, 2009.

[30] M. Medhi, A. Dandautiya, and J. L. Raheja, "Real-Time Video Surveillance Based Structural Health Monitoring of Civil Structures Using Artificial Neural Network," J. Nondestruct. Eval., 2019.

[31] A. L. HODGKIN and A. F. HUXLEY, "A quantitative description of membrane current and its application to conduction and excitation in nerve," J. Physiol., no. 117, pp. 500-544, 1952.

[32] R. Jolivet, T. J. Lewis, W. Gerstner, R. Jolivet, T. J. Lewis, and W. Gerstner, "Generalized Integrate-and-Fire Models of Neuronal Activity Approximate Spike Trains of a Detailed Model to a High Degree of Accuracy," J. Neurophysiol., no. 92, pp. 959-976, 2004.

[33] Q. Yu, H. Tang, K. C. Tan, and H. Yu, "A brain-inspired spiking neural network model with temporal encoding and learning," Neurocomputing, 2014.

[34] A. Mohemmed, S. Schliebs, S. Matsuda, and N. Kasabov, "Method for training a spiking neuron to associate input-output spike trains," in IFIP Advances in Information and Communication Technology, 2011.

[35] S. M. Bohte, J. N. Kok, and H. La Poutre, "SpikeProp : Backpropagation for Networks of Spiking Neurons ErrorBackpropagation in a Network of Spik- ing Neurons," Esann, no. May, pp. 419-424, 2000

[36] M. Amin, M. Shakir, A. Javed, M. Hassan, and S. A. Raza, "Lowcost fault tolerant methodology for real time MPSoC based embedded system," Int. J. Reconfigurable Comput., vol. 2014, 2014. S. F. Wirtz, A. P. A. Cunha, M. Labusch, G. Marzun, S. Barcikowski, and D. S. Id, "Development of A Low-Cost FPGA-Based Measurement System for Real-Time Processing of Acoustic Emission Data: Proof of Concept Using Control of Pulsed Laser Ablation in Liquids," Sensors 2018, 18(6), 1775, vol. 18, no. 6, p. $1775,2018$. 